\title{
Appropriate prescribing in general practice: development of the indicators
}

Of all the activities that take place in general practice, prescribing has the greatest potential to produce health benefits or to cause harm. Therefore, indicators of appropriateness of prescribing should have a central place in evaluating the performance of general practitioners and encouraging improvements in the quality of care. It is surprising, therefore, that little research has been done on the validity and reliability of indicators of prescribing in general practice. In this volume of Quality in Health Care, Cantrill et $a l^{1}$ consider this issue by describing the development and evaluation of several indicators for use in United Kingdom general practice. The study provides an important contribution to the literature in this field and it is likely that the approach taken will have relevance in other countries.

There have been many attempts to develop and use indicators of the appropriateness of prescribing in general practice. ${ }^{2}{ }^{3}$ Indeed it has been suggested that up to 400 different prescribing indicators have been developed by the various health authorities in England and Wales (Roberts, personal communication). There are good reasons for the development of indicators. Firstly, good prescribing is an important issue in quality of care ${ }^{4}$ Secondly, there are costs associated with poor prescribing, including side effects and interactions that might be avoided by more judicious practice. Thirdly, without examining general practitioners' prescribing in the finest detail, it is necessary to have proxies to give an indication of performance.

To be useful, indicators should possess several attributes. ${ }^{5}$ For example, they should cover elements of practice that are acknowledged to be important and not simply record what is easy to measure. Ideally they should be devised with the help of general practitioners, and they should fairly reflect their efforts to prescribe appropriately. The data on which indicators are based should be valid and reliable, and the results should be presented in a user friendly way. It should be made clear that indicators should be used for guidance: they "cannot on their own, provide 'definitive' evidence of success or failure and should be used to raise questions, not provide answers".

The problem with most of the indicators that have been developed for assessing prescribing by general practitioners is that they do not possess all of these attributes. Many indicators focus on cost while paying little attention to other areas of appropriateness. ${ }^{2}$ In other cases, there is an emphasis on basing indicators on what is measured easily, ${ }^{6}$ rather than what is most important. In some cases general practitioners have been involved in the development of indicators. For example, Bateman et al describe how, with prescribing analysis and costs (PACT) data, a consensus group of eight general practitioners set standards for 13 aspects of prescribing. ${ }^{3}$ These indicators can give an impression of prescribing performance and they are based on reliable data that are easily accessible in England and Wales. However, there is no link between PACT data and the reason for individual prescribing decisions, and therefore they have limitations.

The indicators developed by Cantrill et al represent an advance in the evaluation of the appropriateness of general practitioners' prescribing. They have been developed as a result of expert input (with the nominal group technique) followed by a Delphi process involving community pharmacists and general practitioners. The reproducibility of this approach has been questioned. ${ }^{7}$ However, it would seem that the indicators suggested are based on consensus, and they have a reasonable degree of face validity and content validity. Also, the authors have shown which of the suggested indicators are feasible for use on general practitioners' records. Finally, the reliability of the indicators between raters has been assessed. This approach means that we can be reasonably sure that the proposed indicators do cover elements of prescribing that are acknowledged to be important by general practitioners. Also, the approach has shown that some proposed indicators may need to be abandoned if they cannot be applied properly or if reliability between raters is poor. This strengthens arguments for ensuring that the development and use of potential indicators should be accompanied by a process of rigorous evaluation.

It is unlikely that we will ever have a perfect set of indicators of the appropriateness of prescribing by general practitioners, and those suggested in this issue have some drawbacks. For example, collection of prescribing information from patients' general practice records is time consuming and requires expert knowledge. Also, although it is reasonable to base indicators on written records, there are limitations to this approach: improvements in performance may be the result of better recording (which is good in itself) rather than safer and more effective prescribing.

Nevertheless, the indicators suggested ${ }^{1}$ have the potential for further development. For example, they could be incorporated into computerised record systems in general practice. This might reduce the time taken to record the indicators and address problems associated with reliability between raters. Also, where computerised decision support data are available, they could be used as prompts to encourage appropriate prescribing.

The indicators proposed ${ }^{1}$ sit towards one end of a range. At the other end are a host of indicators based on PACT data. These are easy to collect, but may provide relatively poor proxies for the appropriateness of prescribing. It may be that each type of indicator has its place. However, the important issue is that indicators are properly evaluated so that we can be confident about what they are measuring.

ANTHONY J AVERY

Senior Lecturer, Division of General Practice, The Medical School,

University Hospital, Nottingham NG7 2UH, UK. Telephone 0044115 9709387; fax 0044115 9709389; email tony.avery@nottingham.ac.uk

1 Cantrill JA, Sibbald B, Buetow S. Indicators of the appropriateness of long term prescribing in general practice in the United Kingdom: consensus development, face and content validity, feasibility, and reliability. Quality in Health Care 1998;7:130-5.

2 Audit Commission. A prescription for improvement: towards more rational prescribing in general practice. London: HMSO, 1994

3 Bateman DN, Eccles M, Campbell M, et al. Setting standard of prescribing performance in primary care: use of a consensus group of general practitioners and application of standards to practices in the north of England. Br f Gen Pract 1996;46:20-5.

4 Barber N. What constitutes rational prescribing? BMF 1995;310:923-5.

5 Likierman A. Performance indicators: 20 early lessons from managerial use. Public Money and Management 1993;13:15-22.

6 NHS Executive. The new NHS modern and dependable: a national framework for assessing performance (consultation document). Leeds: NHSE, 1998.

7 Shekelle PG, Kahan JP, Bernstein JS, et al. The reproducibility of a method to identify the overuse and underuse of medical procedures. $N$ Engl $f$ Med 1998;338:1888-95. 